Copyright (C) 2014 IEEE. Personal use of this material is permitted. Permission from IEEE must be obtained for all other uses, in any current or future media, including reprinting/republishing this material for advertising or promotional purposes, creating new collective works, for resale or redistribution to servers or lists, or reuse of any copyrighted component of this work in other works. 


\section{A Hardware Implementation and Analysis of Testing MPPT Algorithms on PV Modules}

\author{
Derryn Harvie \\ Dept. Electrical and Computer Engineering \\ Curtin University \\ Perth, Australia
}

\author{
Dr. Sumedha Rajakaruna \\ Dept. Electrical and Computer Engineering \\ Curtin University \\ Perth, Australia
}

\begin{abstract}
Maximum Power Point Tracking (MPPT) algorithms are an important part of photovoltaic systems, with a large amount of literature having been published on different approaches and comparisons. Yet there have been very few practical comparisons between the performances of different algorithms published. This work attempted to resolve this by designing and implementing a custom test system with high accuracy and speed to test algorithms in real time at update rates currently being used in industry. The outcome of the research showed difference in the $P V$ modules tested was more significant than the expected difference between algorithms. However the complete test system performed to specification and is now repurposed for use in teaching and other research.
\end{abstract}

Index Terms-Maximum power point trackers, Photovoltaic systems, Test equipment

\section{INTRODUCTION}

Maximum Power Point Tracking is an important part of PV systems due to the strong influence on the overall system conversion efficiency and economic return. Much has been written on MPPT algorithms in literature with new variations of algorithms continuing to be published $[1,2]$.

The majority of literature published on MPPT algorithms provides extensive mathematical evidence and modeling to demonstrate the strengths and weaknesses of existing and proposed algorithms. Typically this simulation is completed with a combination of step transient simulations, steady state tracking error simulations, and simulations using previously recorded insolation data. A model is then used for the PV array and the desired dynamic insolation conditions are used to control this model to evaluate the MPPT algorithm performance [3].

Whilst this type of simulation provides highly repeatable results, which allow fast and easy comparison of different algorithms, there is a general lack of published robust experimental results to validate the simulated results in practical situations. As a result there is very limited literature on the implementation challenges facing the majority of the recently published algorithms.

In this paper the design and implementation of a test system is presented that was purposely developed to provide a robust experimental test bed for comparing the performance of different algorithms using a PV array.

\section{REVIEW OF EXISTING EXPERIMENTAL METHODOLOGY}

Experimentally testing maximum power point tracking algorithms is very difficult owing to the number of variables inherent in the power conversion from solar energy to the end product, electrical energy at a given voltage or current level. Because of this, there is no single widely used experimental method for the evaluation of the MPPT algorithms.

The most significant variable present is the changing insolation levels that occur in nature. Whilst this is a key part of what MPPT algorithms need to be tested against, it also means that a testing period from one time to the next will never be identical. One solution to this is to use a controlled environmental test chamber [4]. This may be the most appropriate method for testing of physically small PV arrays however this does not scale easily for larger instillations. The PV array itself can be removed as a variable, instead simulating the PV array component and just implementing the hardware MPPT scheme [5]. Whilst this is a valid approach for testing the hardware implementation of the MPPT algorithm against results from modeling, it does not add any significant benefit beyond the complete modeling implementation as the results should be identical if implemented correctly.

This approach can be modified by practically implementing the converter and maximum power point tracker in hardware, but with a PV array simulator [6]. Lastly, the multiple MPPT algorithms can be run in parallel at the same time to remove the dependence on insolation changes [7].

Whilst parallel testing of multiple MPPT algorithms appears to be the most accurate and practically correct solution, the difficulty this introduces is the comparative differences between PV modules. However this method has been used in a number of cases, e.g. [8].

These methods present varying degrees of closeness to the practical usage scenario. Of the methods investigated, the use of an electronic load on multiple panels in an array showed the most flexibility, whilst also having the potential to obtain accurate results. The statistical techniques discussed in [7] present a further path for evaluating the data on parallel arrays once collected. 


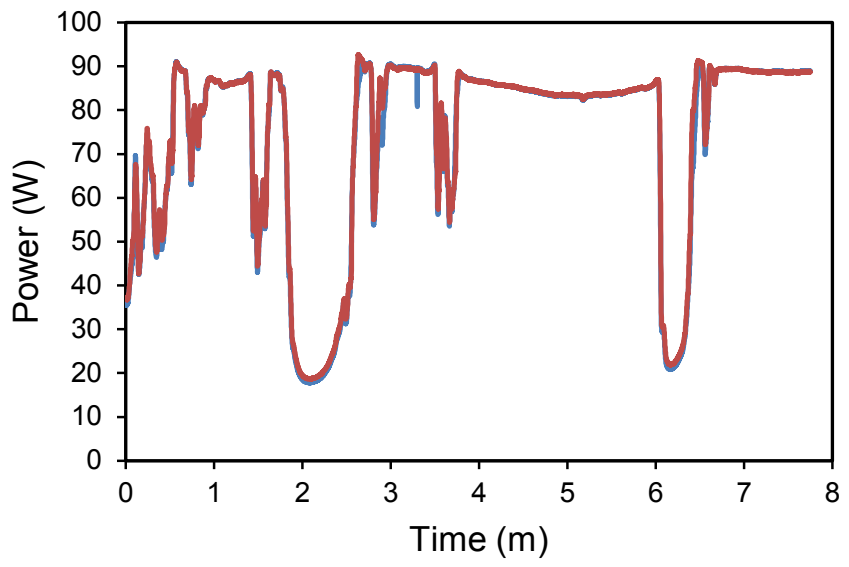

Fig. 1. Power delivered in testing of two P\&O algorithms with an update rate of $20 \mathrm{~Hz}, 250 \mathrm{mV}$ step size (red) and $10 \mathrm{mV}$ step size (blue). The difference in energy integrated over this time period was $0.7 \%$.

\section{SySTEM DESIGN REQUIREMENTS}

In the process of the literature review, it was noted that very few articles discussed their laboratory test systems in detail, their accuracy, speed requirements, or how their test system influenced the results that they achieved. This made the determination of requirements for a test system to be somewhat difficult, as the test system was also not something typically utilized by any industry. Therefore the approach taken was to develop a set of basic requirement based off the best information that was available.

The system was designed around two arrays that were available, the first consisting of Suntech $175 \mathrm{~W}$ monocrystalline modules and the second having lower powered Solarex MSX60 60W poly-crystalline modules. The rating of the higher powered module was used as the input power rating per channel for the system.

The voltage and current resolution requirements were determined from the expected differences in algorithm performance and the MPP voltage and current for the lower powered module. From [6], a Perturb and Observe (P\&O) algorithm and an Incremental Conductance algorithm were compared showing a difference of $1.3 \%$. To confirm this expected magnitude, a prototype electronic load test system was rapidly prototyped. Using the Solarex array with two $\mathrm{P} \& \mathrm{O}$ algorithms having $250 \mathrm{mV}$ and $10 \mathrm{mV}$ step sizes, data was captured during rapidly changing irradiance conditions, shown in figure 1. The power was integrated over the captured time and a difference of $0.7 \%$ was observed between the two implementations. It was deemed a reasonable expectation that the system would need to quantify differences in power between the channels of less than $1 \%$. In order to provide valid results, an absolute accuracy an order of magnitude better than this $(0.1 \%$ or $+/$ $20 \mathrm{mV}$ and $+/-25 \mathrm{~mA}$ ), and a resolution another order of magnitude finer than the absolute accuracy $(0.01 \%$ or $2 \mathrm{mV}$ and $2.5 \mathrm{~mA}$ ) was chosen for the system requirements.

As there is a strong correlation of performance vs step time, a minimum step time becomes a very important factor in the evaluation of perturbative MPPT algorithms [3]. In a system with a DC-DC converter, the settling time of the DC-DC converter control loop is typically the limiting factor. An application note from Microchip ${ }^{\mathrm{TM}}$ was used as a practical example for this loop time [9]. This application note discusses the implementation of a MPPT system using Microchip $^{\mathrm{TM}}$ microcontrollers, with a DC-DC converter PID loop frequency response of $1 \mathrm{kHz}$. It was chosen to implement the MPPT loop speed at $1 / 25$ th of this, $40 \mathrm{~Hz}$. This was used as the loop update rate requirement for the system.

Requirements summary:

- Input power - $175 \mathrm{~W}$ max, $55 \mathrm{~V}, 10 \mathrm{~A}$ per channel

- $\quad$ Algorithm loop rate $-40 \mathrm{~Hz}$

- Absolute accuracy - 20mV, 25mA

- $\quad$ Resolution $-2 \mathrm{mV}, 2.5 \mathrm{~mA}$

\section{IMPLEMENTATION OPTIONS}

Three implementation options were investigated, purchasing commercial off the shelf (COTS) electronic loads, modified COTS electronic loads or producing custom hardware.

\section{A. Commercial Off the Shelf Electronic Load}

This presented the quickest path to a final test system as no custom hardware would be required. The MPPT algorithms would be calculated on a PC, and the updated load point transferred back to the electronic load. This implementation has been present previously e.g. [10].

A number of commercial offerings were evaluated. In terms of input power, accuracy and resolution, meeting the specifications was easily achieved by a number of products. However the timing specifications with respect to changing of the load point, then receiving back a valid voltage and current reading was not specified for any of the loads evaluated.

Schematics and parts lists were available for some of the available products such as the Agilent ${ }^{\mathrm{TM}}$ 6060, and the ADC used in the BK precision 8500 is publically available knowledge. By assessing the capabilities of these parts, it was found they could not provide the sample rates at the resolution required. Therefore it was determined that those loads would not be capable of meeting the timing specification required. This ruled out the direct use of COTS electronic loads.

\section{B. Modified COTS Electronic Load}

The option of modifying a commercial electronic load to meet all of the specifications was investigated for one of the cheaper loads on the market. The Maynuo ${ }^{\text {TM }}$ M9700 series was considered as it could be purchased economically enough to build a multi-channel test system. It was determined a modification kit could be built consisting of a daughterboard with new microcontroller, ADC, PC interface and firmware. This would have been a significant irreversible modification and likely would not have included supporting the standard front panel interface and display. 
This was deemed to be a feasible option, with the benefit of the mechanical rack mount enclosure design being complete. A multichannel system could be built using a number of modified loads in a rack enclosure.

\section{Full Custom Hardware}

Building the hardware system from ground up provides increased flexibility of tailoring the system to meet the end objective. It does however increase the design and build time to realize a working system, and in this case was calculated to give only a minor reduction in total system cost. However ultimately it was deemed the most practical option as it avoided potential logistics issues and allowed building a system that would utilize all six individual modules available in the Solarex PV array. It also avoided having to engineer around legacy interfaces, components and physical constraints. Figure 2 shows a high level overview of the system designed and constructed for each channel, except the processor, $\mathrm{AD}$ and $\mathrm{DA}$ converters are shared across two load channels. This is a very similar system design as was implemented in all the commercial electronic loads evaluated.

\section{LOAD CELL TOPOLOGY}

Three options were considered for implementing the load cell:

- A boost converter to vary the power into a battery or resistive load.

- Pulse Width Modulate a fixed resistive load with an LC input filter.

- A fully linear variable load using power transistors, as in figure 3.

The linear load cell topology shown in figure 3 was used, the primary advantage over the other two options is the lack of any switching noise. This made the analog front end for the ADC simpler with excellent noise performance being achieved with no filtering. It also has the advantage that significantly higher bandwidth can be achieved due to the lack of an inductor in the current path or switching cycle delay. While this is not a primary consideration for this application, it makes the load more flexible by being able to generating $\mathrm{AC}$ load currents for other testing applications.

It does however make dissipating the levels of power required more expensive as the MOSFET devices themselves take all the input power (rather than a battery or cheap resistive load.) To solve this problem, economical heatpipe based heatsinks designed for cooling desktop computer CPUs were used to provide high performance cooling to the load MOSFETs. This made for a relatively complex mounting arrangement, with two T03P FETs mounted in a reverse fashion with adhesive to the supporting PCB. The heatsink is then clamped down on top of the FET tabs in the same manner that it would normal attach over a CPU. Using Thermaltake Contac 21 CPU coolers this was tested successfully with $175 \mathrm{~W}$ continuous power input.

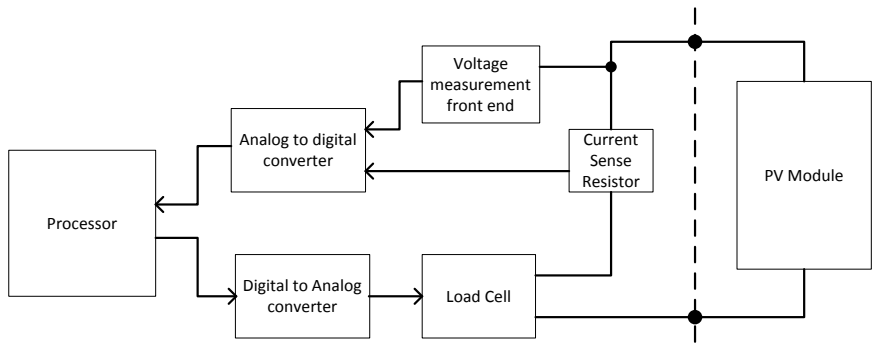

Fig. 2. High level hardware architecture of implemented MPPT testing electronic load. The processor calculates the MPPT algorithm and transmits the operating point back to a PC for logging.

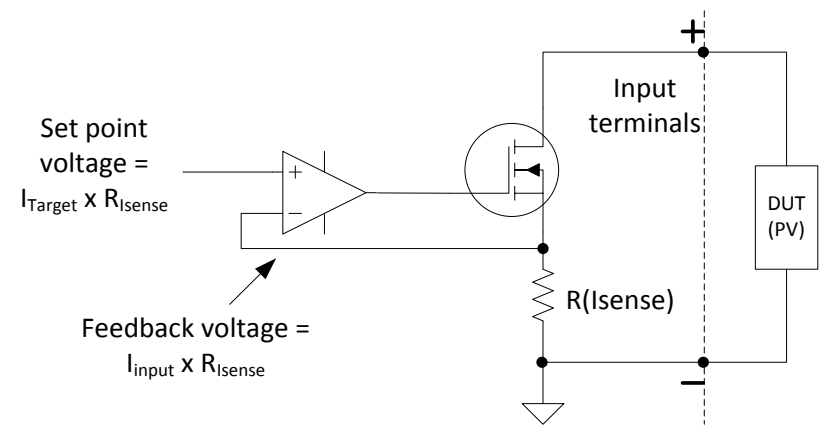

Fig. 3. Opamp-Mosfet load cell topology used in the electronic load, duplicated twice per channel for increased current and power handling.

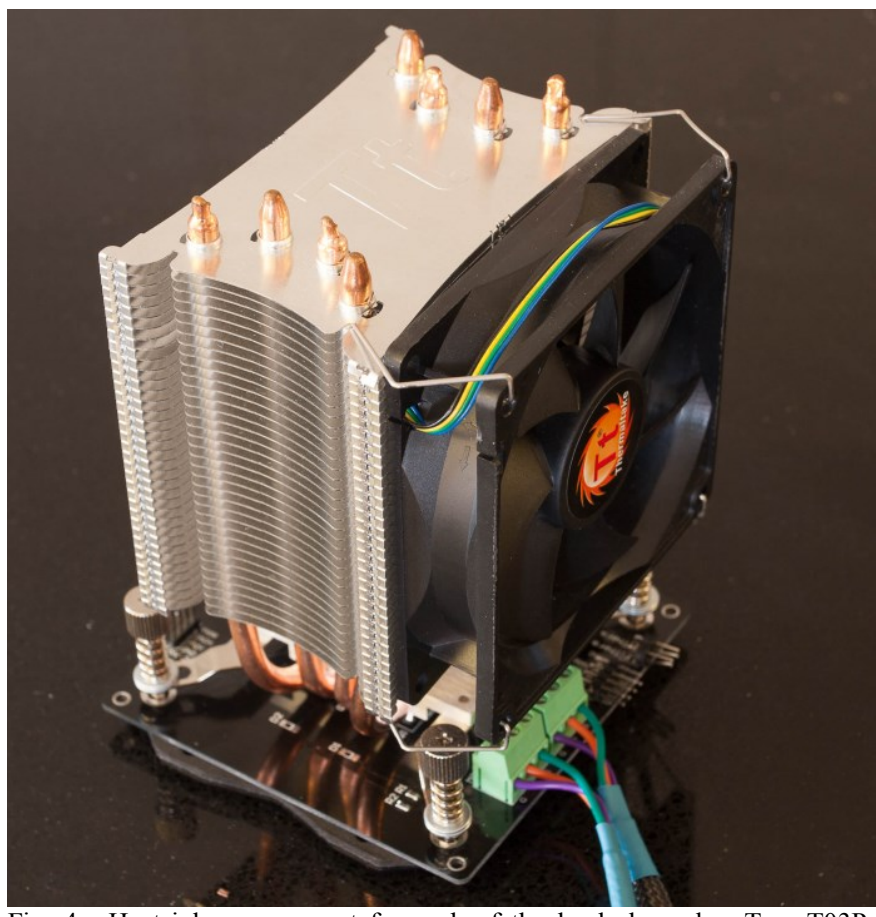

Fig. 4. Heatsink arrangement for each of the load channels. Two T03P MOSFETs are mounted upside down on the PCB with the PC CPU heatsink clamped down on top with thermal grease.

As maximum power point tracking typically uses voltage control rather than current control [3], the load cells needed to be controlled in terms of voltage. Tests were completed implementing this outer control loop in software to simplify the hardware implementation. However it was found the 
$\mathrm{ADC}$ and DAC resolution was not fine enough, nor could the loop speed be implemented fast enough to achieve the desired result. Instead the current control loop was enclosed in a switchable voltage control loop. The switching is completed using analog multiplexers to allow switching between current control and voltage control in software.

\section{System COMPONENT CHOICE}

The microcontroller chosen for the application was of ARM $^{\text {TM }}$ M4 architecture, which had the advantage of hardware floating point and easily available development tool chain. Having fast floating point support on the microcontroller made the coding of different algorithms much faster than working in fixed point, or having the overhead of using a PC to perform the calculations. This was coupled on each control board, which controls two load channels, with a Maxim ${ }^{\mathrm{TM}}$ MAX11045 6-channel 16bit parallel sampling ADC. This part has an external pin that triggers the end of sampling phase, allowing for all measurements of current and voltage across all load boards to be coordinated simultaneously. The DAC used on each board to control the load cells is a Texas Instruments ${ }^{\mathrm{TM}}$ DAC8552 16bit two channel, again to provide simultaneous update to the two load channels on each control board.

However the timing of the DAC updates is less critical than the voltage and current measurement, as the load cells must be allowed sufficient time to settle prior to any feedback from the change being taken. With the V and I measurement, any time skew between channels can affect the result if the irradiance changes between the measurements on each channel. Communication back to the PC used for data logging and providing overall coordination of long test sequences was completed using the on-chip Full Speed USB interface of the microcontroller. This allowed hosting of the data and control of the test sequences remotely via LAN connected to the PC.

\section{TEST RESULTS}

Due to the total power difference in algorithm performance having been observed to be very small, it was important to validate the test hardware on the PV arrays. Characterization of the panels within the two arrays was completed with unsatisfactory results in all cases. Tests on all the PV modules were completed by sweeping the load voltage from zero to open circuit in parallel to obtain comparative IV characteristics for the modules in the array.

The array consisting of Solarex modules was found to have three out of six modules in a degraded state, with significantly lower power output than the $44 \mathrm{~W}$ NOCT rating. The three serviceable modules were significantly closer to the original power rating, however there was still a non-linear difference of greater than $1 \%$ across the most significant portion of the IV characteristic (either side of the MPP).
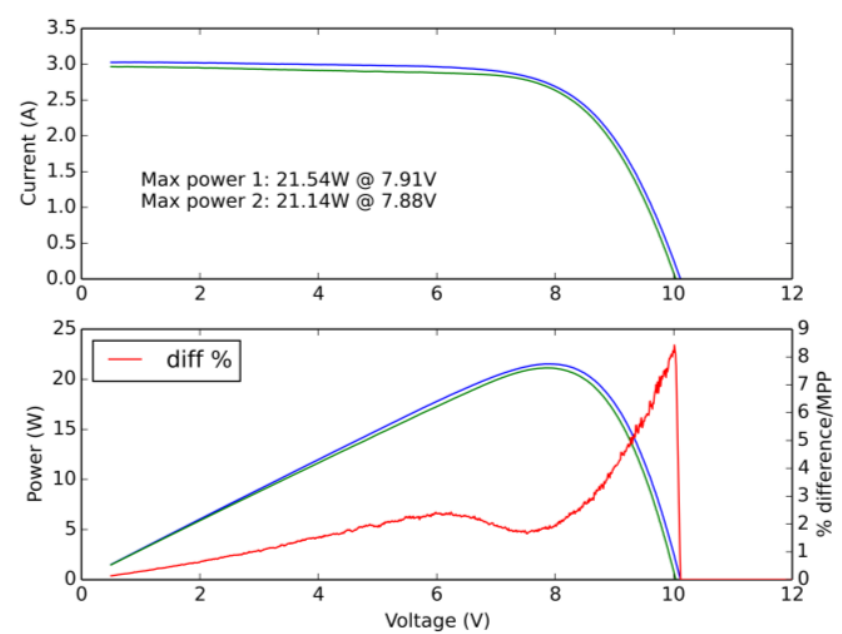

Fig. 5. IV sweep of a single Solarex module's individual strings for matching. The power difference is relative to the MPP power of the first string.
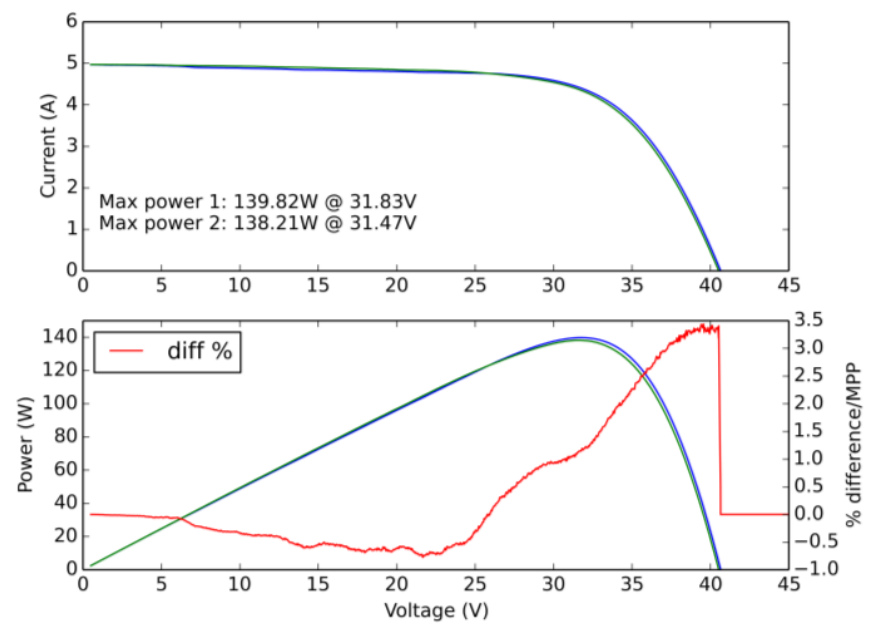

Fig. 6. IV sweep of two modules from the second array. The power difference is relative to the MPP power of the panel 1.
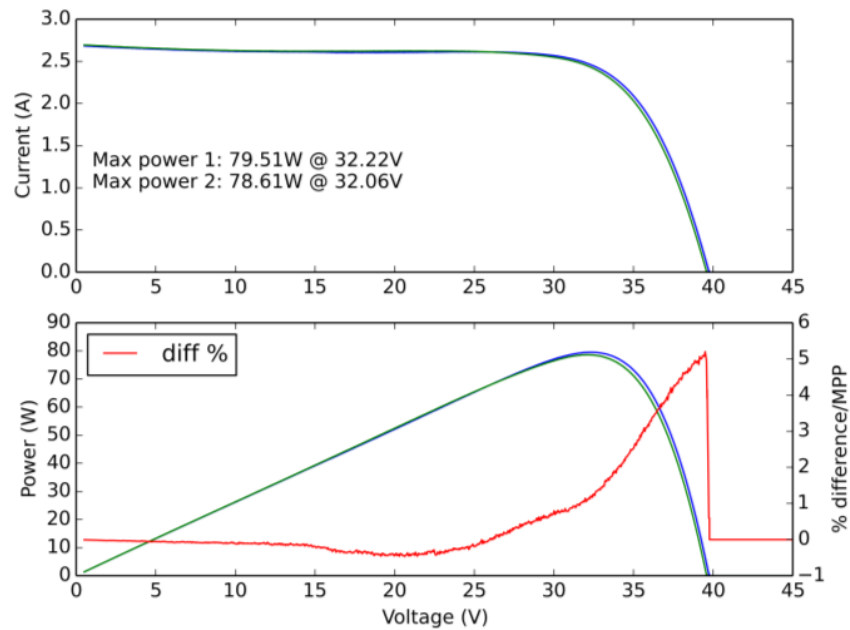

Fig. 7. IV sweep of two modules from the second array tilted away from the sun. 


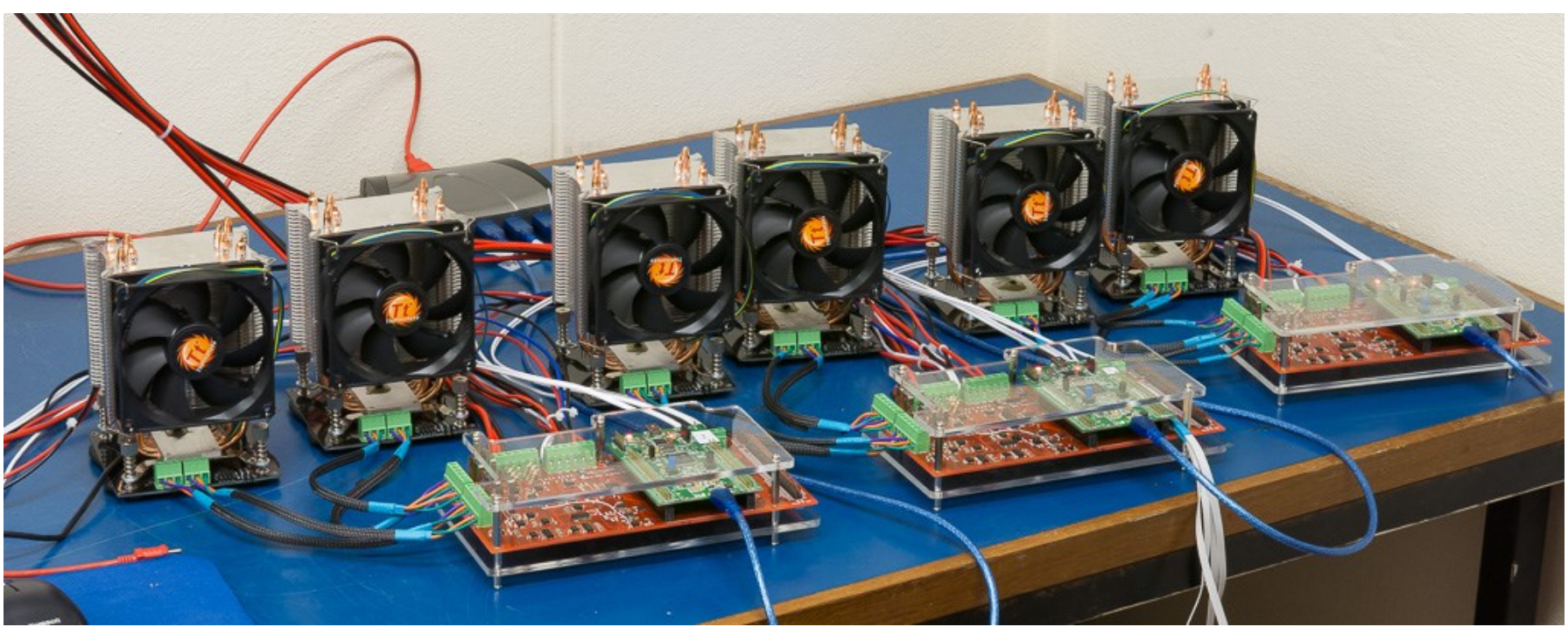

Fig. 8. Complete bench test system of six load channels with heatsinks connected to a PC for logging via USB. The load channels are connected through to a PV array of individual panels fixed mounted to the building roof.

Two Solarex modules that had been stored for the majority of time since manufacture were tested for matching between individual strings. This potentially offered the best chance for matching as the strings of cells had been exposed to the same environmental conditions. The results from one test are shown in figure 5, where it can be seen the difference between strings is more significant than the likely difference between algorithms. The second panel that was tested showed differences between the strings of a similar magnitude.

Panels from the larger array were tested in a similar manner and the results shown in figure 6. This array is much newer, with the panels being installed for approximately 18 months.

The matching of these panels was much better, particularly very close to the MPP. However the differences were still too large in the context of the differences in algorithms. To further explore the differences the same tests were conducted with the panels rotated away from the sun, the result in figure 7 . The relative differences between the panels were observed to shift with changes in irradiance, which made any simple attempts to calibrate out the difference in the panels ineffective.

\section{CONCLUSION}

Accurate comparison of multiple MPPT algorithms in a practical setting is difficult due to the differences in panels. A significant amount of testing was conducted during this work in an attempt to produce valid results to practically compare algorithms. The test system was built to specifications that exceed the expected differences in algorithm performance by an order of magnitude to ensure that would not be a factor. However, profiling of different panels and strings within panels showed in every case that the differences between panels or strings were more significant that the differences in the algorithms performance.

Attempts were made to use simple correction factors, to calibrate out the differences between panels. It was found the differences were highly non-linear with changes in irradiance and temperature, rendering the calibration of the panels unable to produce the confidence levels required.

The direct comparison of algorithms running in parallel, on un-matched panels proved to be too inaccurate to be useful in long term power output testing. However it may be possible for panels to be carefully matched from large quantities of stock to provide the level of matching required. This would require significant resources to compare cells, and would require monitoring of the cell matching as they aged.

From this research the testing of MPPT algorithms on PV panels under ambient conditions for overall output power can practically only be achieved with limited accuracy.

\section{REFERENCES}

S. Lyden, M. E. Haque, A. Gargoom, and M. Negnevitsky, "Review of Maximum Power Point Tracking approaches suitable for PV systems under Partial Shading Conditions," in Power Engineering Conference (AUPEC), 2013 Australasian Universities, 2013, pp. 1-6.

[2] A. Talha, H. Boumaaraf, and O. Bouhali, "Evaluation of maximum power point tracking methods for photovoltaic systems," Archives of Control Sciences, 2011.

[3] N. Femia, G. Petrone, G. Spagnuolo, and M. Vitelli, Power Electronics and Control Techniques for Maximum Energy Harvesting in Photovoltaic Systems: Taylor \& Francis, 2012.

[4] P. Wolfs and Q. Li, "Hardware Implementation and Performance Analysis of a Current Sensor Free MPPT for High Performance Vehicle Solar Arrays," ed, 2007.

[5] G. Petrone, G. Spagnuolo, and M. Vitelli, "A Multivariable Perturb-and-Observe Maximum Power Point Tracking Technique Applied to a Single-Stage Photovoltaic Inverter," Industrial Electronics, IEEE Transactions on, vol. 58, pp. 76-84, 2011.

[6] D. P. Hohm and M. E. Ropp, "Comparative study of maximum power point tracking algorithms using an experimental, programmable, maximum power point tracking test bed," in Photovoltaic Specialists Conference, 2000. Conference Record of the Twenty-Eighth IEEE, 2000, pp. 1699-1702.

[7] X. Weidong, H. H. Zeineldin, and Z. Peng, "Statistic and Parallel Testing Procedure for Evaluating Maximum Power Point Tracking 
Algorithms of Photovoltaic Power Systems," Photovoltaics, IEEE

Journal of, vol. 3, pp. 1062-1069, 2013.

[8] U. Zimmermann and M. Edoff, "A Maximum Power Point Tracker for Long-Term Logging of PV Module Performance," IEEE $J$.

Photovolt., vol. 2, pp. 47-55, 2012.

[9] S. O. Mihnea Rosu-Hamzescu, "AN1521 Practical Guide to Implementing Solar Panel MPPT Algorithms," 2013.

[10] G. Carannante, C. Fraddanno, M. Pagano, and L. Piegari, "Experimental Performance of MPPT Algorithm for Photovoltaic Sources Subject to Inhomogeneous Insolation," Industrial Electronics, IEEE Transactions on, vol. 56, pp. 4374-4380, 2009. 